\title{
Intuitive 3D Role Playing Game Development Using Unreal Development Kit (UDK)
}

\author{
Syung-Og An ${ }^{1}$, Man-Geun Park ${ }^{2}$, Shin-Jin Kang ${ }^{3}$ and Soo-Kyun Kim ${ }^{1}$ \\ ${ }^{1}$ Department of Game Engineering, Paichai University \\ ${ }^{2}$ Dragonfly Company, Seoul, Korea \\ ${ }^{3}$ School of Games, Hongik University \\ Corresponding Author: Soo-Kyun Kim (kimsk@pcu.ac.kr)
}

\begin{abstract}
The release of various mobile game engines and other easy-to-use game engines have opened up an era wherein anyone from beginner to expert can easily develop games. Currently launched smart phones have large screens, clear and high-definition displays, and high-performance chipsets. This allows 3D Role Playing Games, previously only available on the PC, on a mobile phone. The Unreal Development Kit (UDK) is highly suitable for designing high-quality games and provides the perfect environment to design games on mobile devices.

This paper explains an easy method for intuitive development of $3 D$ RPG games. In order to design the game intuitively, $3 D$ geographic models and characters were designed firsthand using Zbrush and 3D MAX, and cinematic pictures were inserted by actively using the Kismet provided by UDK to allow the viewers to watch movie-like event images during the game.
\end{abstract}

Keywords: Kismet, Unreal Development Kit, Game Engine, Role Playing Games

\section{Introduction}

As the game industry rapidly grows, game development programs and game engineering skills are also becoming more and more technical and diverse. New game development programs wherein anyone from beginner to expert can use are constantly being released, and now anyone can easily develop games. The Unity 3D engine provides an intuitive interface that solved various difficulties in game design. Using this game engine $_{2}$ many game developers are designing various mobile games. The current internal supply rate of smart phones in South Korea is at a world-class level of 94\%.

The fierce competition between smart phone manufacturers have led to several advancements of the smart phones such as large screens, clear and high-definition displays, and high-performance chipsets, and ultimately allowing the game execution environment to be similar to that of the PC. Taking these factors into consideration ${ }_{2}$ it will not be too long until high-specification PC games will be allowed on smart phones. The users will soon lose interest in the currently popular easy and cute games and demand more high-definition and large scale games. According to the Korean Creative Content Agency, the average development time for PC game is 20.3 months.

However, judging by the development speed of smart phones, PC games will be allowed on smart phones perhaps within one year, or a maximum five years at the latest. The objective of this thesis is to predict the users' needs and design a high-performance game for the mobile devices. For this, the Unreal engine was used as it provides a posh graphic performance. GEARS of WAR is currently the most well-known game developed in UDK environment, and in South Korea the company Dragonfly is developing a mobile version of Special Force 2 using the Unreal engine. 


\section{Formatting Your Paper}

The proposed development objective is to design a 3D RPG game that provides simple operations and diverse contents. The dynamic and lively sense of action applied by remarkable graphic performances, various events, and animation, will provide a game environment that the user can become immersed in for hours. In order to design a 3D game for the mobile, 3D characters and 3D geographic features were used. The 3D modeling process can be divided into character modeling [4] and background modeling [5], and overall includes texture, rigging, and animation production.

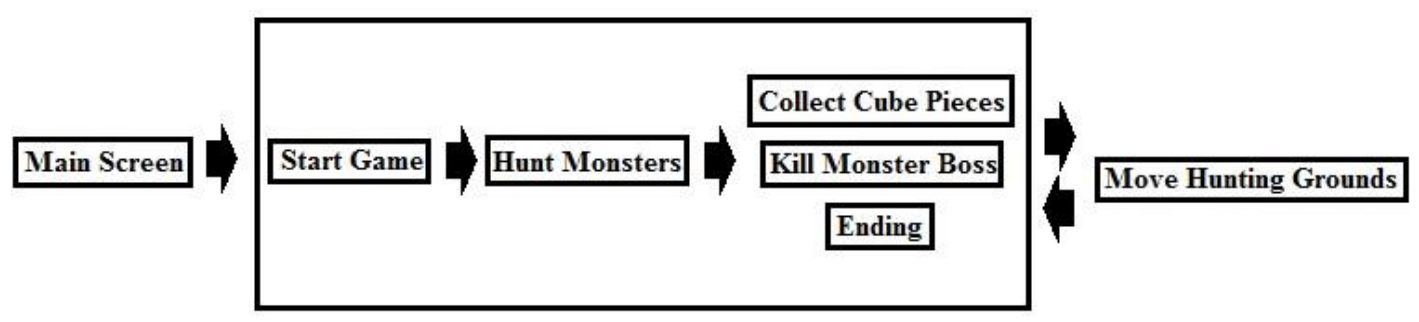

Figure 1. System Structure Map

Figure 1 shows a simple system structure map needed to progress in the game. The overall flow of the game starts at main screen and once the game is started the user must hunt any monsters that appear while collecting cube pieces that can be acquired through hunting, and ultimately seal the gate to defeat the monster boss and win the game.

\section{Model Production and Design}

\subsection{Model production and Geographic Model Design}

In order to develop a 3D model appropriate for this thesis, the raw data reference from the original 3D MAX was exported into Zbrush and transformed into High Polygon modeling. This process is not just a simple 3D operation and needs not only basic knowledge and understanding about art but also multisensory capabilities. In this paper, Zbrush is used to design characters that reflect each of their distinctive features.

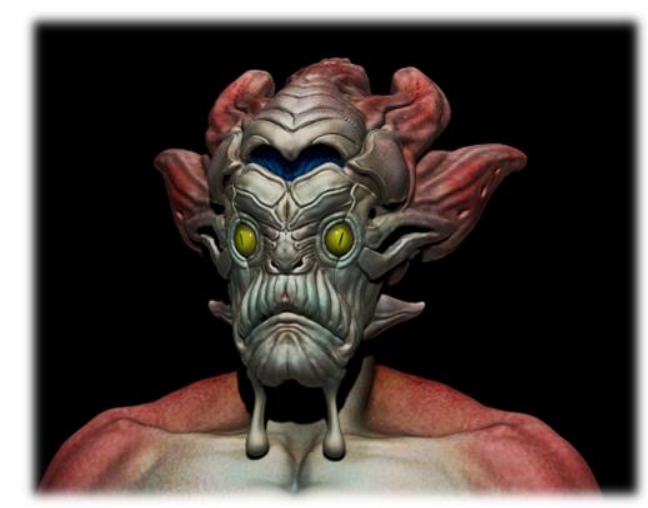

Figure 2. 3D High Polygon Modeling

\subsection{D Geographic Feature Materialization}

For the materialization of geographic features, instead of using the basic features provided by the Unreal engine [7], the geography is modified to fit the scenario. The 
geographic features differ in shape, size, and each sector has different events. A map is formed using the LandScape function, provided in UDK.

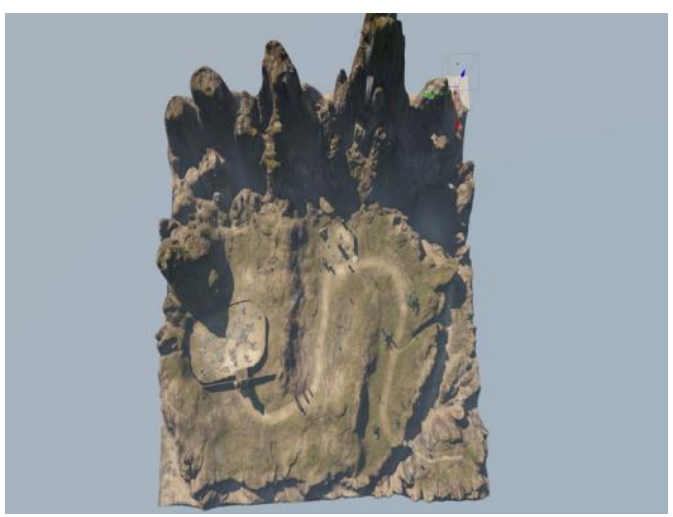

Figure 3. 3D Geographic Feature Production using LandScape

The LandScape feature [2] in UDK only allows preliminary shapes. A separate program called World Machine is used to apply weathering, erosion, and other effects and thus creates a much more brilliant outcome. After this step, the Height Map and Weight Map of the finished geographic model is exported to UDK, where the final touches to texture and forms are made and an objective that fits the geography is installed. Figure 3 demonstrates a geographic feature designed using the LandScape feature and World Machine.

\subsection{Nomal Map Formation}

A Normal Map formula is the value of depth expressed in the actual height, and allows for a quality that a Low Polygon mash cannot achieve. Normal Map uses Specular Map and Ambient Occlusion Map together, leading to very high quality materials. This is a method often used in high-specification games. The downside is that the diverse and large texture requires massive amounts of data and the production environment must be able to handle the weight.

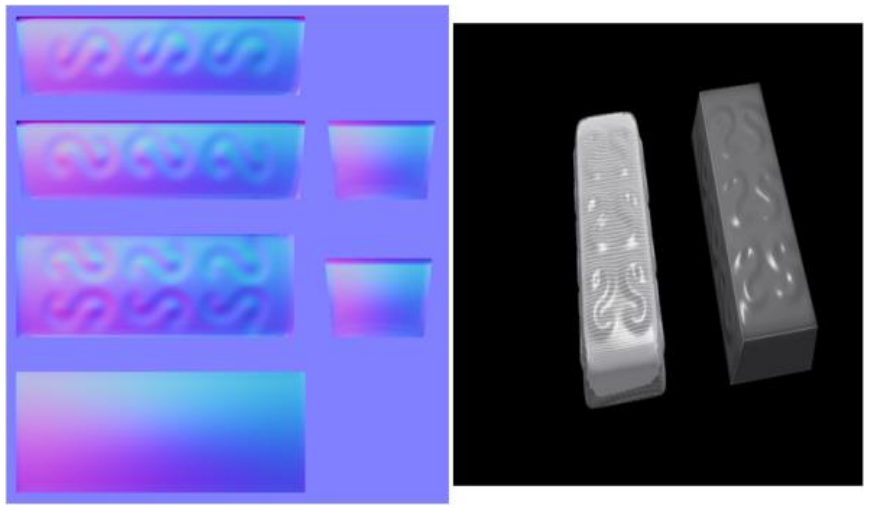

Figure 4. Normal Map Texture Formation

Figure 4 displays a normal map with the texture applied to it. An advantage of this method is that various texture effects can be layered onto a Low Polygon mash, which is useful in optimization.

\subsection{Clothing Production Using Marvelous Designer}

As shown in Figure 5, Marvelous Designer is a clothing simulation program that is actually used in the fashion industry. The clothing production mimics the real-life 
tailoring process and demonstration can be given by simulation. This feature has not been used very long in games, but once there is a more in-depth understanding of tailoring technique, there will be more room for competitiveness. After learning the techniques, it is easy to dress up the character in any type of clothing, whether basic or complicated. Clothing production is not limited to the character and can also be used to decorate the background such as on curtains, blankets, carpets, and many more.

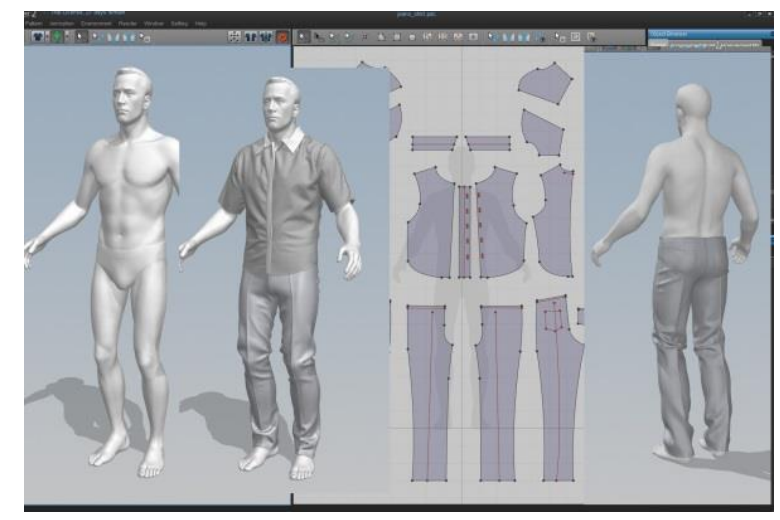

Figure 5. Marvelous Designer

\subsection{SSS (Subsurface Scattering) Shader Application}

Subsurface Scattering, as shown in Figure 6, is when the light does not completely reflect off the surface such as human skin or plant stems, and is partially absorbed - thus the light penetrates with a scattering effect. The SSS Shader is perfect for creating realistic human bodies because it expresses this type of subsurface scattering effect. However, it has a complicated structure and it could be difficult to achieve good results without vast knowledge about subsurface scattering.

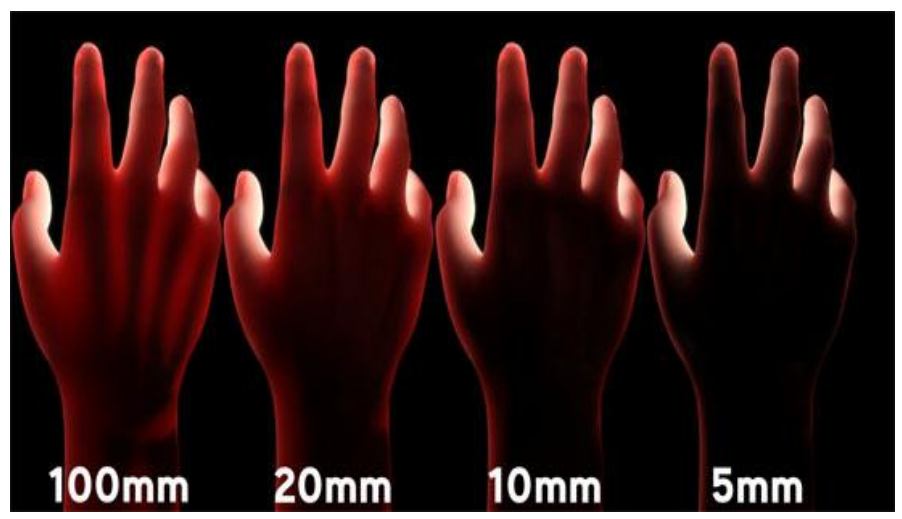

Figure 6. Subsurface Scattering

\section{Game Design Using UDK}

\subsection{High Visual Game Graphics}

In this thesis, High Polygon modeling, various textures and shaders were applied to provide visual delight to the user, as shown in Figure 7 below. All the different types of effects also amplify the visual aspect. These visual elements make the game really stand out from the rest. The player's perspective of geographic features formed by high altitude landscapes presents a large, whole new world for the user. 


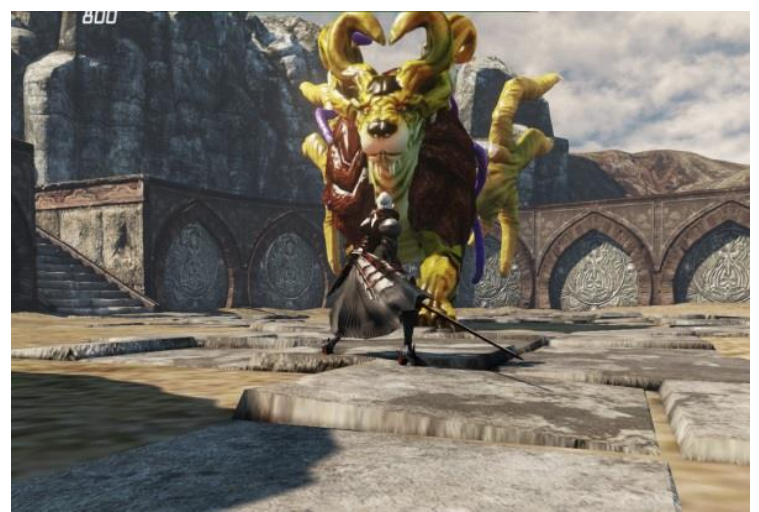

Figure 7. Game Graphic Design and Application

\subsection{Event Effects Using Kismet}

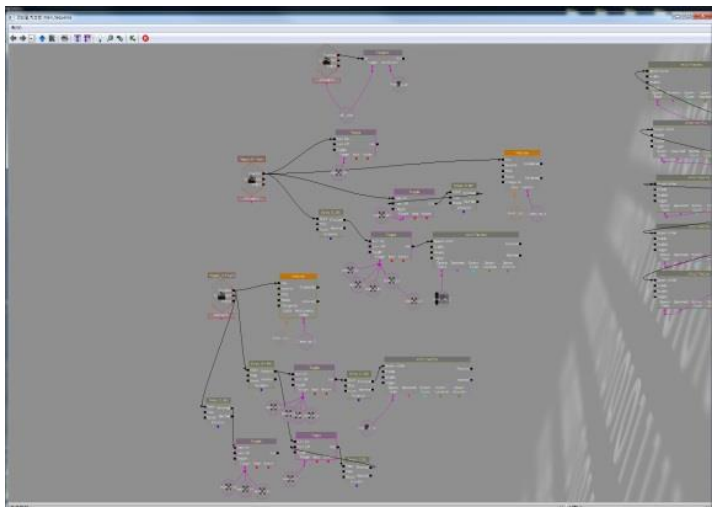

Figure 8. Kismet Composition

As shown in Figure 8, UDK supports the Kismet System. Kismet is responsible for all event construction within a game, and is often used in RPG games that usually contain several complicated events. Biggest advantages are that anyone could understand the progress of the events at first glance and that any problems during the event process can easily be detected. Settings can adjust anything from camera angles to sound, object arrangement, movement, etc. Furthermore, events can be set up so that a new one comes up after certain requisites are met during the game.

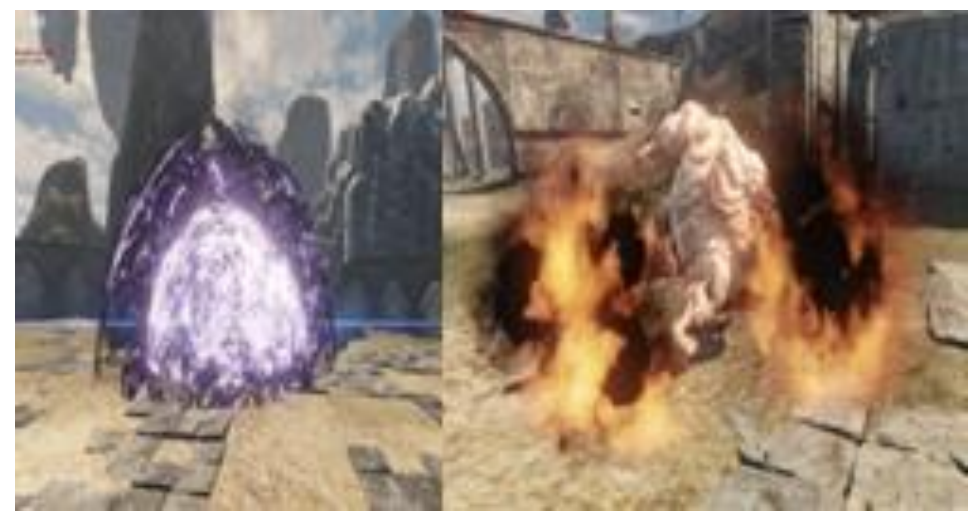

Figure 9. Cinematic Picture

Figure 9 shows a cinematic picture created by using the Kismet System and camera effects. During the game the user is able to watch the event in motion picture through this. 


\subsection{Various Motions and Monsters}

Many artificial intelligence monsters with fluid movement appear in this game. These monsters start approaching the user when he/she comes within a certain range and attack once in contact with the user. This is a useful feature that creates tension and absorbs the user into the game. Also, as shown in figure 10, the various different ranks of monsters from regular to boss [6] will provide ongoing interest.

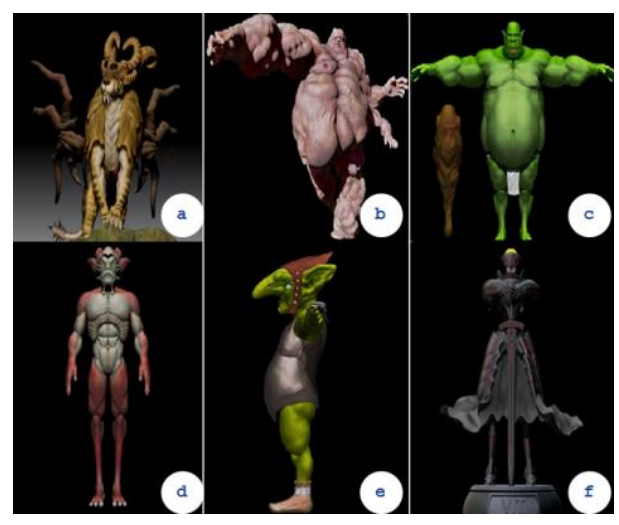

Figure 10. Diverse Characters

In Figure 10, (a) is the monster boss Behemoth, (b) is the mid-boss Abomination, (C) is an ogre, (d) is a giant, (e) is a goblin, and $(f)$ is the main character Rachel.

\section{Developmental Environment and Testing}

This game was developed under the following environment: hardware DGA device Radeon 70 2GB RAM, and operating system Windows 7 using the Unreal Script library from UDK version 3.

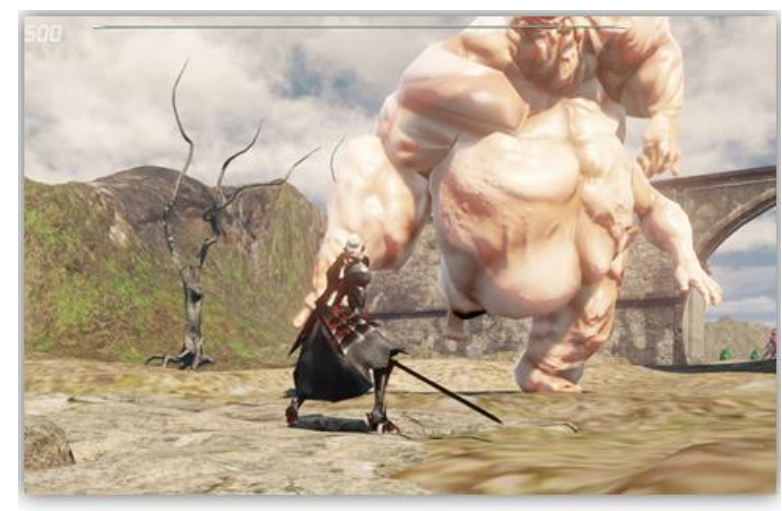

Figure 11. Battle Scene 


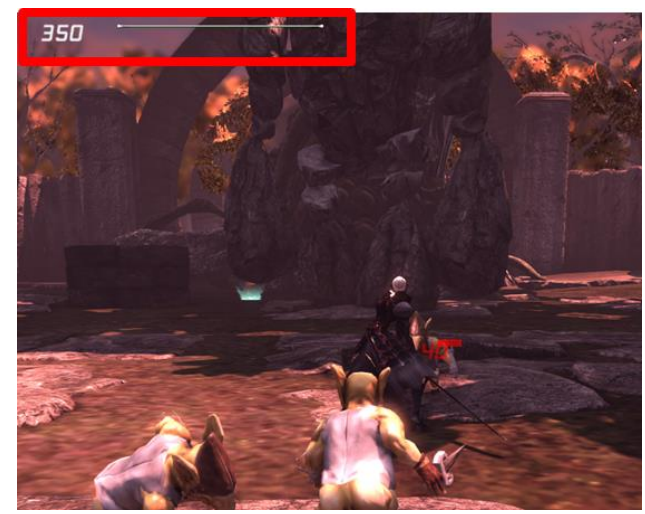

Figure 12. Energy Bar

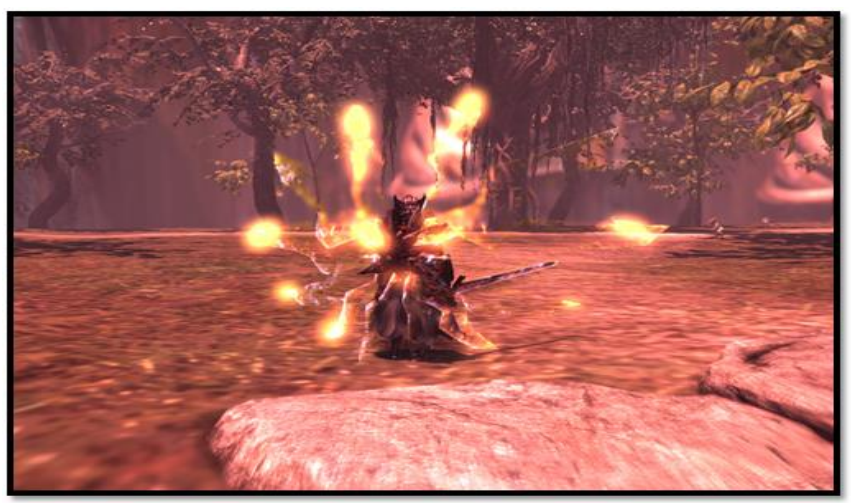

Figure 13. Monster Animation

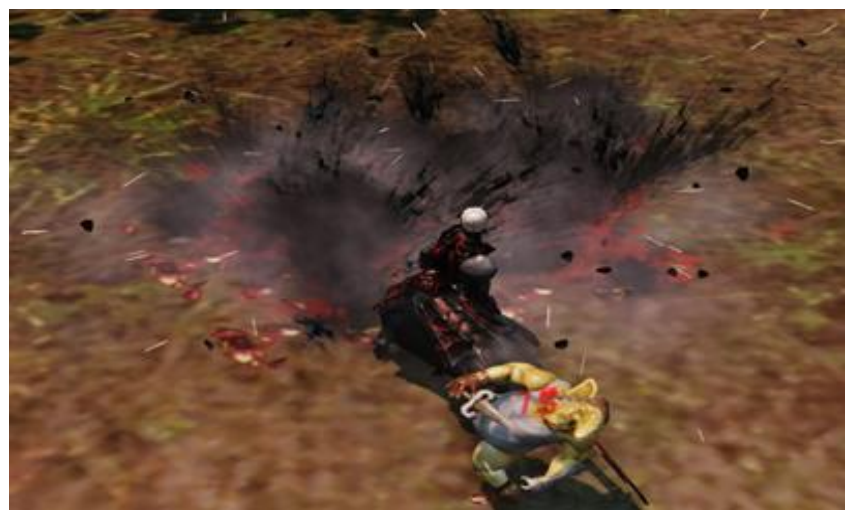

Figure 14. Character Transformation

Figures 11 and 14 show the game in action tested on a PC. Figure 11 shows the midboss Abomination approaching the player using its artificial intelligence. Monsters are programed to attack whenever the character falls within a specified range. Figure 12 displays the energy bar that turns up during battle to indicate the monster's remaining strength. Energy bars for boss rank monsters are programmed to appear on the top of the screen. Figure 13 demonstrates the explosion of a monster after being defeated by the user's attacks. The animation effects increase the liveliness and satisfaction of the game. Figure 14 shows a character transformation, a feature that can be activated by obtaining transformation items during the game. 


\section{Conclusion}

To allow intuitive development of the game in this thesis, 3D geographic feature models and characters were directly designed using Zbrush and 3D MAX. The thesis also explains how to easily design a 3D RPG game. Remarkable results were shown by using not only just basic tools from Photoshop, but also advanced tools from Marvelous Designer and 3D-COAT. Unreal Development Kit's extensive data management capacity, massive contents, and available resources really broadened the game developing process.

In future research, all current UDK functions must be applied in order to design a game not only plausible in high-performance mobile, but also low-performance mobile as well.

\section{References}

[1] Alan T., "UDK Game Development," Cengage Learning PTR; 1 edition, December 30, (2011).

[2] Hyungho J., "3D background graphics production notes (Korean edition)," Sung Andang, (2012).

[3] Eric K., "Introducing ZBrush 3rd Edition," Sybex; 3rd edition, June 5, (2012).

[4] Leo H., "The Art of Game Characters," Harper Design, June 14, (2005).

[5] Thomas M., "Unreal Development Kit Game Design Cookbook," Pack Publishing, February 22, (2012).

[6] Jason B., "Mastering Unreal Technology: The Art of Level Design," Sams, December 12, (2004).

[7] Jason B., Zac P., Jeff W., "Mastering Unreal Technology, Volume I: Introduction to Level Design with Unreal Engine 3," Sams Publishing, (2009).
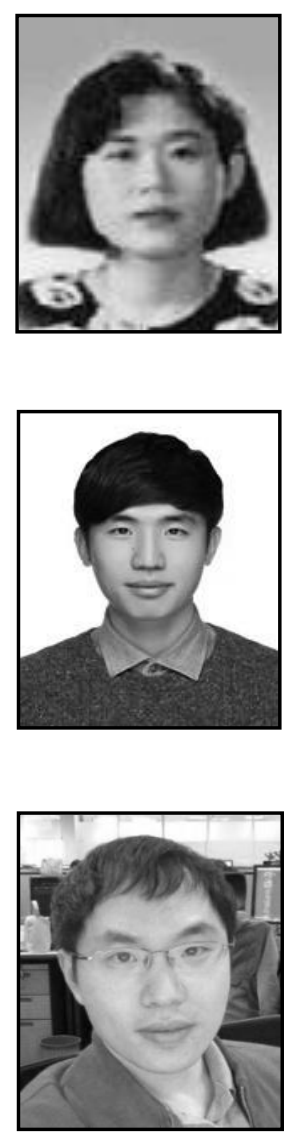

\section{Authors}

Syung-Og An, she is a professor in the Department of Game Engineering at Paichai University, Korea. She received M.S. and Ph.D. in Computer Science \& Engineering Department of Korea University, Seoul, Korea, in 1984 and 1989, respectively. She was a visiting professor at the University of Pennsylvania State University from 1993 to 1994. Her research interests include multimedia system, computer graphics and database.

Man Geun Park, he graduated from Department of Game Engineering in 2015. He has worked at the 3D modeler and Game programmer of Dragonfly Company.

Shin Jin Kang, he received Ph.D. degree in Computer Science from Korea University in 2011. Since 2003, he has worked at Sony Computer Entertainment Korea and NCsoft as a lead game designer. He is now a professor at the School of Games at Hongik University. He is also the technical advisor of NCsoft. 


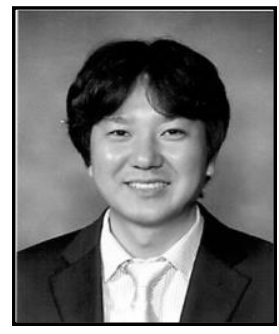

Soo-Kyun Kim (Corresponding Author), he received Ph.D. in Computer Science \& Engineering Department of Korea University, Seoul, Korea, in 2006. He joined Telecommunication R\&D center at Samsung Electronics Co., Ltd., from 2006 and 2008. He is now a professor at Department of Game Engineering at Paichai University, Korea. Dr. Kim has published many research papers in international journals and conferences. Dr. Kim has been served as Chairs, program committee or organizing committee chair for many international conferences and workshops; Chair of ICCCT'11, ITCS'10, HumanCom'10, EMC'10, ICA3PP'10,,FutureTech'10, ACSA'09, Em-Com'09, CSA'09, CGMS'09, ISA'09, SIP'08,FGCN'08 and so on. Also Dr. Kim is guest editor of the International Journal of "IET Image Processing" and "Multimedia Tools and Applications". His research interests include multimedia, pattern recognition, image processing, mobile graphics, geometric modeling, and interactive computer graphics. He is a member of ACM, IEEE, IEEE CS, KACE, KMMS, KKITS and KIIT.

Corresponding Author: Soo-Kyun Kim (kimsk@pcu.ac.kr) 
International Journal of Multimedia and Ubiquitous Engineering

Vol.10, No.6 (2015) 